\title{
Finite current stationary states of random walks on one-dimensional lattices with aperiodic disorder
}

\author{
Hiroshi Miki \\ Research Institute for Humanity and Nature, \\ 457-4 Motoyama Kamigamo, Kita-ku, Kyoto, 603-8047, Japan
}

July 11, 2018

\begin{abstract}
Stationary states of random walks with finite induced drift velocity on one-dimensional lattices with aperiodic disorder are investigated by scaling analysis. Three aperiodic sequences, the Thue-Morse (TM), the paperfolding (PF), and the Rudin-Shapiro (RS) sequences, are used to construct the aperiodic disorder. These are binary sequences, composed of two symbols A and $\mathrm{B}$, and the ratio of the number of As to that of Bs converges to unity in the infinite sequence length limit, but their effects on diffusional behavior are different. For the TM model, the stationary distribution is extended, as in the case without current, and the drift velocity is independent of the system size. For the PF model and the RS model, as the system size increases, the hierarchical and fractal structure and the localized structure, respectively, are broken by a finite current and changed to an extended distribution if the system size becomes larger than a certain threshold value. Correspondingly, the drift velocity is saturated in a large system while in a small system it decreases as the system size increases.
\end{abstract}

Keywords: random walk, aperiodic disorder, stationary states, scaling, multifractal 


\section{Introduction}

A random walk on a lattice, or more generally on a complex network, is a simple stochastic process which describes a classical transport phenomenon in a real space or a step-by-step state change in an abstract state space. Due to and in spite of the simplicity of the process, various and nontrivial properties have been found and analyzed in detail, both mathematically and physically [1, 2].

In the presence of disorder, i.e., where the hop probability or rate from one site to another is not uniform, the behavior of the random walk is strongly modified, especially in lower dimensions, not only quantitatively but also qualitatively. When the disorder is random and uncorrelated, various methods have been developed for analysis. Especially, from methods based on renormalization group, many results, some of which are exact, have been obtained [3, 4, 5, 6. One of the most remarkable results is so-called ultraslow diffusion, where the diffusion is strongly suppressed by disorder, and the averaged mean-square displacement grows extremely slowly, i.e. on a log-time scale [8, 9]. Correspondingly, the stationary state on a finite lattice is strongly localized.

Systems with aperiodic disorder are also interesting for investigation. An aperiodic disorder is generated by a certain set of deterministic rules but does not have any periodicity. It is this point that distinguishes aperiodic from random uncorrelated disorder. Moreover, aperiodic disorder is considered to be intermediate between uniformity or periodicity and random disorder. Hence, the study of systems with aperiodic disorder is probably a good first step toward understanding systems with more general correlated disorder. Generally, it is difficult to construct an aperiodic disorder with desirable characteristics. Fortunately, for one-dimensional lattice systems various aperiodic disorders can be easily constructed with the help of the aperiodic sequences which have been investigated mathematically. Note that in addition to the theoretical and mathematical interest, systems with aperiodic disorder have been fabricated artificially and investigated experimentally[10]. As expected, some results that are unique for aperiodically disordered systems have been obtained. One of the most remarkable is the appearance of anomalous diffusion, where the mean-square displacement grows slowly - less than linearly with time [7]. Correspondingly, a singular stationary probability distribution with a remarkable hierarchical structure appears [11].

If the hop rates do not satisfy a certain condition (see Eq.(7)), a finite drift velocity is induced, at least in a finite system, and in the stationary state a finite current flows through the lattice. As is well known, a finite current forces the stationary distribution to be extended. Therefore, it is an interesting problem to investigate how the stationary distribution without current will be changed by the presence of a finite current - particularly when the distribution is localized or singular. In the present paper we use scaling analysis to cope with this problem for the cases of lattices with aperiodic disorder. As in our previous study 11, we consider the aperiodic disorders constructed by the Thue-Morse (TM), paperfolding (PF), and RudinShapiro (RS) sequences. These three aperiodic sequences have several common properties: (i) They are binary sequences, which are composed of two types of symbols, A and B. (ii) They are constructed systematically from initial sequences and by iteration of specific substitution rules.

(iii) The ratio of the number of As to that of Bs converges to unity in the infinite sequence length limit. Nevertheless, these aperiodic disorders have different effects on the diffusional behavior [7] and correspondingly on the stationary probability distribution[11, since they have difference wandering exponents (see Sec. 2.2).

We focus on the dependence of the drift velocity and the localization structure of the stationary probability distribution on the system size. In order to characterize the latter, we use multifractal analysis[12], as in our previous study[11]. This approach has been applied to characterize the scaling structure of distributions in various systems, including those of the energy dissipation in turbulence[13, 14], the sidebranch structure of dendrites [15], and the quantum localization problem[16], where the localization property of the wavefunction is studied. 
The organization of the rest of this paper is as follows: In Section 2, we formulate our model and method for analysis. We describe our one-dimensional random walk, give the expressions of the observables, and introduce the aperiodic sequences from which the disorder is constructed. Then we describe the method of the multifractal analysis for the distribution on a one-dimensional support, the criterion for localization and the finite-size effect. In Section 3 , we present our results and a discussion. Section 4 is dedicated to our conclusion and future outlook.

\section{Model and method}

\subsection{Random walk on one-dimensional disordered lattice}

Let us consider a random walk on a one-dimensional lattice with only nearest neighbor hopping allowed. This process is described by the master equation:

$$
\frac{\partial p_{j}(t)}{\partial t}=w_{j-1, j} p_{j-1}(t)+w_{j+1, j} p_{j+1}(t)-\left(w_{j, j-1}+w_{j, j+1}\right) p_{j}(t)
$$

where $p_{j}(t)$ is the probability for the particle to be on site $j$ at time $t$ and $w_{j, k}$ denotes the hop rate for the particle from site $j$ to $k$. We impose the periodic boundary condition $p_{j+L} \equiv p_{j}$ and $w_{j+L, k+L}=w_{j, k}$, where $L$ denotes the system size, the number of sites on the lattice. Interestingly this master equation is known to be equivalent to the transverse-field Ising model[7].

We construct the disorder according to an aperiodic binary sequence, $S$, composed of two types of symbols, $A$ and $B$. For example, let us take $S=A B A A B A A B A B \cdots$. For this sequence, the hop rates are assigned as:

$$
w_{j, j+1}=1, \quad \text { for all } j,
$$

and

$$
w_{j+1, j}= \begin{cases}a, & \text { the } j \text {-th symbol of } S \text { is } A, \\ b, & \text { the } j \text {-th symbol of } S \text { is } B .\end{cases}
$$

At least as far as we are concerned with the stationary state, the assignment does not lose generality, since the quantities related to the stationary state, the probability distribution and the drift velocity, are expressed as a function of the ratio $w_{j, j+1} / w_{j+1, j}[9]$.

For the stationary state, $d p_{j} / d t=0$, the exact expression of the probability distribution is obtained in ref. [9] as:

$$
p_{j}=\frac{r_{j}}{\sum_{k=1}^{L} r_{k}}
$$

where

$$
r_{j}=1+\sum_{k=1}^{L-1} \prod_{l=1}^{k} w_{j+l, j+l-1} .
$$

From this stationary probability, the drift velocity is obtained as

$$
v_{d}=\frac{L}{\sum_{j=1}^{L} r_{j}}\left(1-\prod_{j=1}^{L} w_{j+1, j}\right) .
$$

If

$$
\prod_{j=1}^{L} w_{j+1, j}=1
$$

holds, no current exists even in a finite lattice. We call this condition the "zero-current condition". For an infinite lattice, however, $v_{d}$ can vanish even if Eq.(17) does not hold, due to 
the divergence of the denominator of the RHS of Eq.(6). If the zero-current condition does not hold, the finite current tend to force the stationary distribution to be extended throughout the lattice. Trivially, the stationary distribution is extended if the current is so strong that the disorder effect can be considered negligible. We are interested in the regime where the effects of current and disorder are comparable.

\section{$2.2 \quad$ Aperiodic sequences}

We give a brief review of the binary aperiodic sequences which we consider for the disorder. Here we describe the properties relevant to the discussion; the initial sequence, the substitution rules, and the wandering exponent. The readers can refer to the literature, for example, refs. [7, 11, 17, 18] for more details or a general framework of aperiodic sequences.

The Thue-Morse (TM) sequence is systematically constructed from the initial sequence $S_{1}=A B$ and the iterative substitution rules $A \rightarrow A B$ and $B \rightarrow B A$. This sequence is known to be related to the Koch snowflake[19], which is known to be a fractal curve. The length of the sequence of the $n$-th generation, $S_{n}$, is $2^{n}$. The wandering exponent $\Omega$ is defined as

$$
\Delta_{n}(L)=\left|N_{n}(A)-N_{n}(B)\right| \sim L^{\Omega},
$$

where $\Delta_{n}(L)$ is called the geometric fluctuation of the sequence of the $n$-th generation, $L$ denotes the length of the sequence, and $N_{n}(A)$ and $N_{n}(B)$ denote the numbers of symbols $A$ and $B$, respectively. This exponent characterizes how the effect of the disorder changes with the system size and plays a crucial role in the diffusional behavior. For the TM sequence, $\Omega=-\infty<0$, and thus the effect of the disorder decreases as the system size increases. The diffusional behavior is, even in the case without current, essentially similar to that in uniform or periodic systems.

The paperfolding ( $\mathrm{PF}$ ) sequence is generated by the initial sequence $S_{1}=A A$ and the substitution rules $A A \rightarrow A A B A, A B \rightarrow A A B B, B A \rightarrow A B B A$, and $B B \rightarrow A B B B$. This sequence is related to another fractal, known as the dragon curve[20]. The wandering exponent of this sequence is zero. In this case, the geometric fluctuation grows logarithmically with the system size, $\Delta(L) \sim \log L$, and thus the effect of disorder is almost independent of the system size. An anomalous diffusion and a multifractal stationary probability distribution are observed under the zero-current condition[7, 11].

The Rudin-Shapiro (RS) sequence is generated by the initial sequence $S_{1}=A A$ and the substitution rules $A A \rightarrow A A A B, A B \rightarrow A A B A, B A \rightarrow B B A B$, and $B B \rightarrow B B B A$. The wondering exponent of this sequence is $\Omega=1 / 2>0$. In this case, the effect of disorder grows stronger as the system size increases, and it changes the diffusional behavior qualitatively. Without current, a localized stationary probability distribution and ultraslow diffusion are observed. Note that the value of the exponent $1 / 2$ coincides with that of the random sequence.

\subsection{Multifractal analysis}

To characterize the structure of the stationary probability distribution, we use the multifractal analysis method. For a given probability distribution, suppose that the support of the distribution is completely covered with disjoint patches of size $\epsilon$, and let $p_{j}(\epsilon)$ be the measure assigned to the $j$-th patch. The measure is expected to scale locally as

$$
p_{j}(\epsilon) \sim \epsilon^{\alpha_{j}},
$$

where $\alpha_{j}$ denotes the singularity exponent around the $j$-th patch. Moreover, the number of patches taking the value of the singularity exponent between $\alpha$ and $\alpha+d \alpha, N(\alpha)$, is expected to scale as

$$
N(\alpha) d \alpha \sim \epsilon^{-f(\alpha)} d \alpha
$$

where $f(\alpha)$ is, roughly speaking, the fractal dimension of the set of patches taking $\alpha$. 
In our study, aperiodic chains are one-dimensional and given through taking the limit $L \rightarrow \infty$ for the sequence of finite length. Thus we formulate multifractal analysis on a onedimensional finite lattice and evaluate the results of the system of infinite length limit by systematically extrapolating the results of the systems of finite size.

For a given probability measure on a one-dimensional lattice of size $L,\left\{P_{j}\right\}_{j=1,2, \cdots, L}$, the partition function $Z(q, L)$ is introduced as

$$
Z(q, L)=\sum_{j, p_{j} \neq 0}\left(p_{j}\right)^{q}
$$

The multifractal exponent for the finite system, $\tau(q, L)$, is defined as

$$
\tau(q, L)=-\frac{\log Z(q, L)}{\log L} .
$$

The singularity exponent $\alpha=\alpha(q, L)$ and the fractal dimension $f=f(q, L)$ are obtained through the Legendre transformation:

$$
\begin{aligned}
\alpha(q, L) & =\frac{\partial \tau(q, L)}{\partial q}, \\
f(\alpha(q, L)) & =q \alpha(q, L)-\tau(q, L) .
\end{aligned}
$$

Numerically it is better to evaluate them directly, without using numerical differentiation. To do this, we follow the method presented in ref.[13, summarized below.

Let us construct a new probability measure $\left\{\mu_{j}(q)\right\}$ from $\left\{p_{j}\right\}$ as

$$
\mu_{j}(q)=\frac{\left(p_{j}\right)^{q}}{\sum_{j=1}^{L}\left(p_{j}\right)^{q}} .
$$

Then let us define $\zeta(q, L)$ and $\xi(q, L)$ as

$$
\begin{aligned}
\zeta(q, L) & =\sum_{j=1}^{L} \mu_{j}(q) \log p_{j}, \\
\xi(q, L) & =\sum_{j=1}^{L} \mu_{j}(q) \log \mu_{j}(q),
\end{aligned}
$$

From these we obtain $\alpha(q, L)$ and $f(\alpha(q, L))$ as

$$
\begin{aligned}
\alpha(q, L) & =-\frac{\zeta(q, L)}{\log L}, \\
f(\alpha(q, L)) & =-\frac{\xi(q, L)}{\log L} .
\end{aligned}
$$

It is easy to show, by direct calculation, that the definition Eqs.(18) and (19) respectively coincide with Eqs.(13) and (14).

Then the evaluation of finite size effect and extrapolation to the infinite system size limit are carried out. The value of $\alpha(q)$ is defined as the limiting value of $\alpha(q, L)$ as the system size goes to infinity:

$$
\alpha(q):=\lim _{L \rightarrow \infty} \alpha(q, L) .
$$

From Eqs.(18) and (20), we expect

$$
\alpha(q)-\alpha(q, L)=\mathcal{O}(1 / \log L)
$$

From this we can evaluate the value of $\alpha(q)$ from the plot of $\alpha(q, L)$ against $1 / \log L$ and the extrapolation to $1 / \log L \rightarrow 0$. The fractal dimension $f(q)$ is evaluated similarly. 


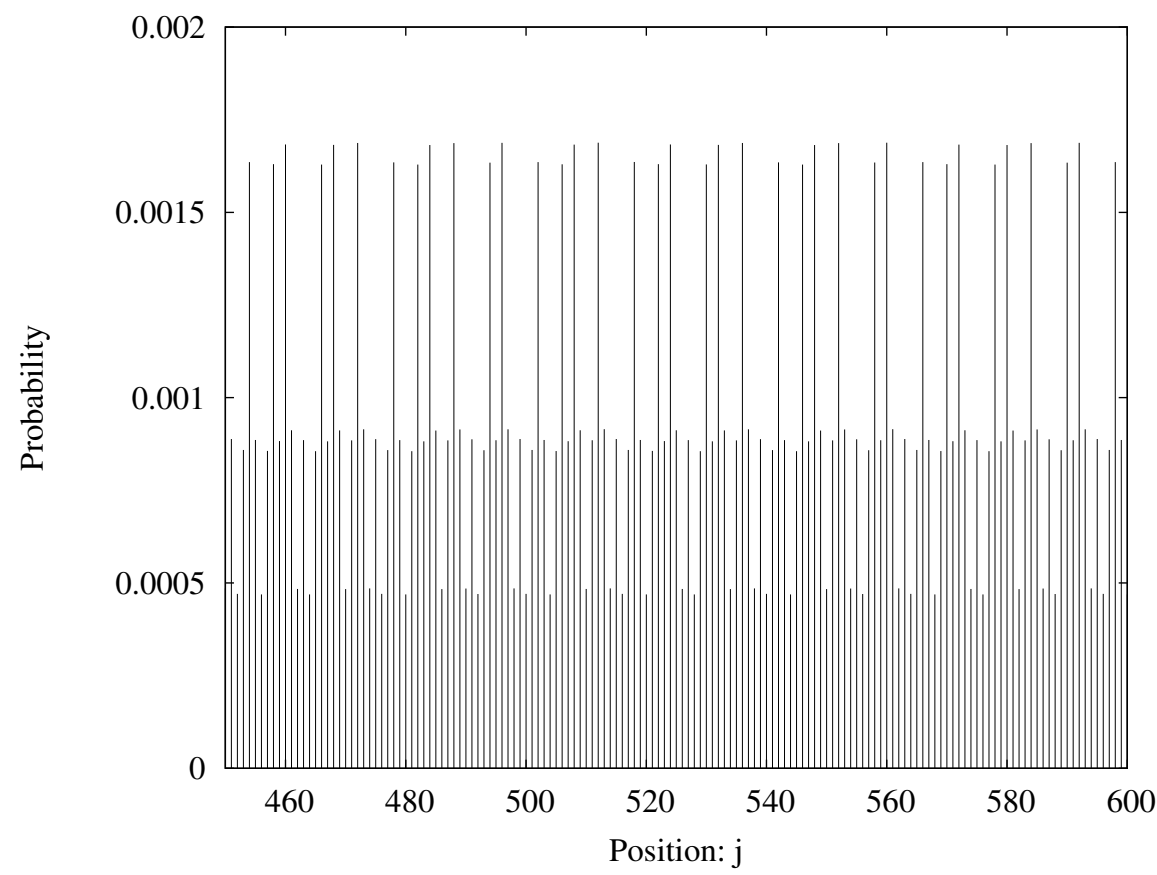

Figure 1: Stationary probability distribution $\left\{p_{j}\right\}$ for the TM model with $a=0.5, b=1.8$, and $L=1024$. Only the region $450 \geq j \geq 600$ is shown for visibility. The distribution is extended.

Let $\alpha_{\min }$ and $f_{\min }$ be $\alpha(q \rightarrow \infty)$ and $f(q \rightarrow \infty)$, respectively. We can read the localization property of a given distribution from these exponents [16]. For an extended distribution, the multifractal spectrum of finite systems, which draws a curve in the $\alpha-f$ plane, converges to a single point, $\alpha=f=1$ in the limit as $L \rightarrow \infty$. For a localized distribution, both $\alpha_{\min }$ and $f_{\min }$ converges to 0 . For a singular distribution, $\alpha_{\min }$ converges to a certain finite value and $f_{\min }$ may converge to 0 or a finite value, depending on the case. In our discussion below, we investigate the scaling properties of only $\alpha_{\min }$, since that is sufficient for determining the localization property.

\section{Results and discussion}

We are interested in the cases where the local preferential hopping direction depends on the disorder. Thus we restrict ourselves to the cases with $a<1, b>1$, and $a b<1$, unless otherwise noted. The condition $a b<1$ means that the current is in the positive direction (at least in the $L \rightarrow \infty$ limit). We expect that the characteristic properties are invariant under $a \leftrightarrow b$, since for the underlying aperiodic sequences, the ratio of the number of As to that of Bs converges to unity in the infinite length limit. We can quantify the degree of the breaking of the zero-current condition as $1-a b$.

\subsection{Thue-Morse(TM) model}

The stationary probability distribution of the TM model is extended even when there is no current[11], since the wandering exponent of this sequence is negative. Therefore, in the case with finite current, the extendedness of the distribution is still maintained, and only a quantitative difference is observed. Figure 1 shows the stationary distribution of the TM model with $a=0.5, b=1.8$, and $L=1024$. The measures are fluctuating around one of the three values, $1 / C, a / C$, and $1 / a C$, where $C=2^{n-1}+\left(a+a^{-1}\right) 2^{n-2}$ and $n=\log _{2} L$, which are the measures in the case without current. 


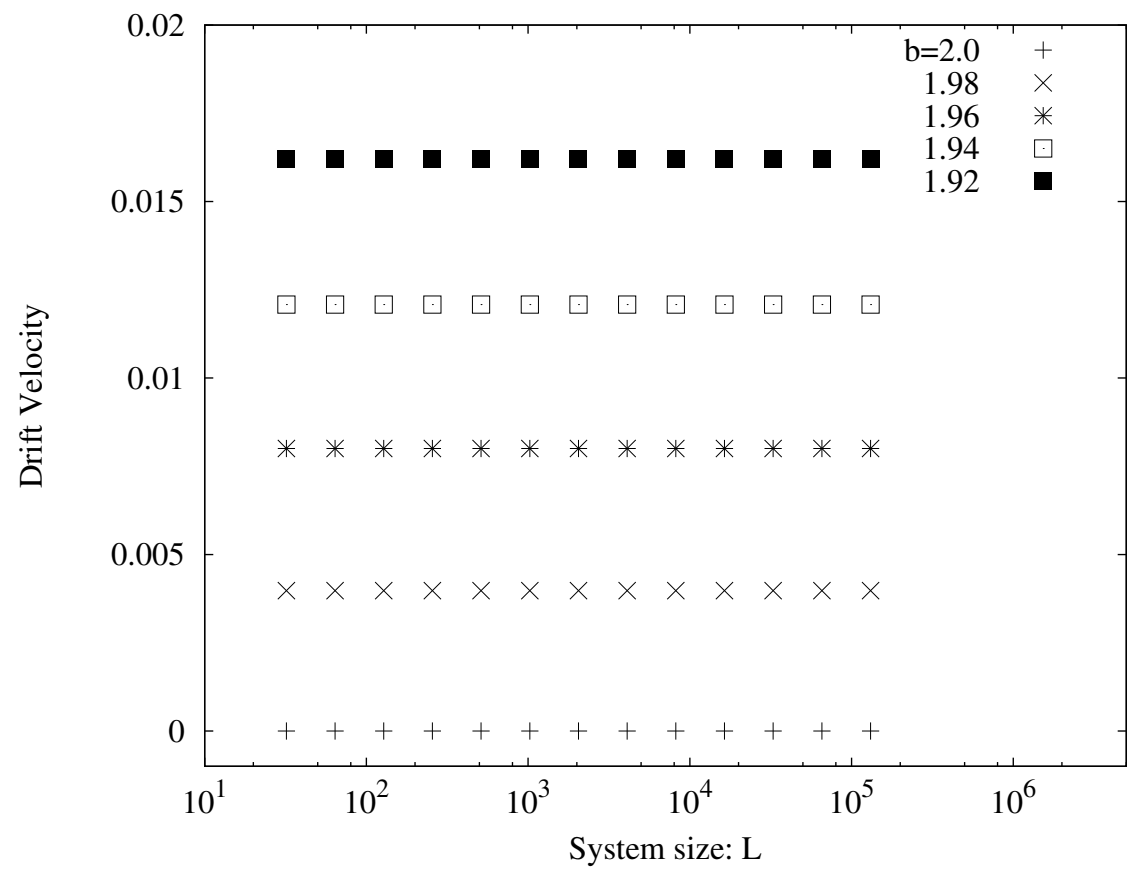

Figure 2: Plots of the drift velocity $v_{d}$ for the TM model for several values of $b$ with $a$ fixed at 0.5 .

The drift velocity is numerically almost independent of the system size. (See Fig,2.) This is the consequence of the negativeness of the wandering exponent and the fact that for the TM sequence, the numbers of $\mathrm{A}$ and $\mathrm{B}$ exactly coincident in any generation $n$. We also note that the drift velocity is proportional to the degree of the breaking of the zero-current condition:

$$
v_{d} \propto 1-a b,
$$

as in cases where the arrangement of $\mathrm{A}$ and $\mathrm{B}$ is periodic.

\subsection{Paperfolding(PF) model}

Before analyzing the structure of the stationary distribution in detail, we consider the dependence of the drift velocity $v_{d}$ on the system size. Figure 3(a) shows this plot for several values of $b$ with $a=0.5$ fixed. First, for $b=2.0$, where the zero-current condition is satisfied in the limit of infinite system size, it is observed that the drift velocity decays algebraically:

$$
v_{d} \sim L^{-\delta}
$$

where the exponent $\delta$ depends on $a$. Figure 3 (b) shows the dependence of $\delta$ on $a$. It converges to unity in the limit as $a \rightarrow 1$, the homogeneous limit.

If the zero-current condition does not hold even in the limit of infinite system size, the drift velocity decays for a small system, and it is saturated and converges to a certain finite value for a large system. Figure 4(a) shows the dependence of the drift velocity on the degree of the breaking of the zero-current condition, $|1-a b|$ with $a=0.5$ fixed. It is observed to be a power-law

$$
\left|v_{d}\right| \sim|1-a b|^{\gamma}
$$

regardless of the sign of $1-a b$. Figure 4(b) shows the dependence of the exponent $\gamma$ on $a$.

For the PF model without current, the stationary probability distribution is called "singular", neither extended nor localized. It has a hierarchical structure and is characterized by multifractal spectrum[11]. Figure 5 s shows the stationary distribution of the PF model with 

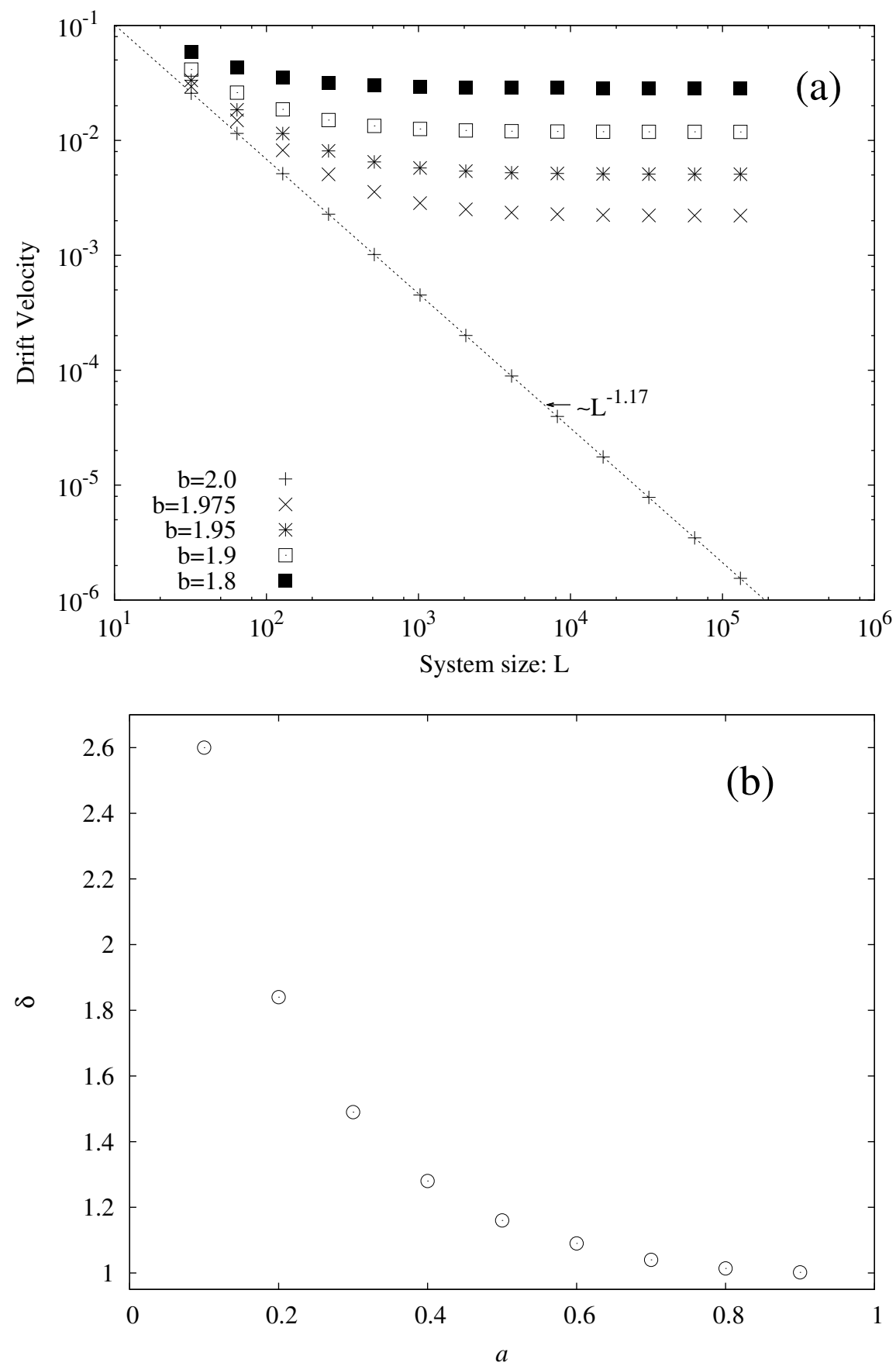

Figure 3: (a) Plots of the drift velocity $v_{d}$ as a function of the system size $L$ for the PF model for several values of $b$ with $a$ fixed at 0.5. (b) Dependence of the exponent $\delta$ (see Eq.(23)) on $a$. It converges to unity in the limit $a \rightarrow 1$. 

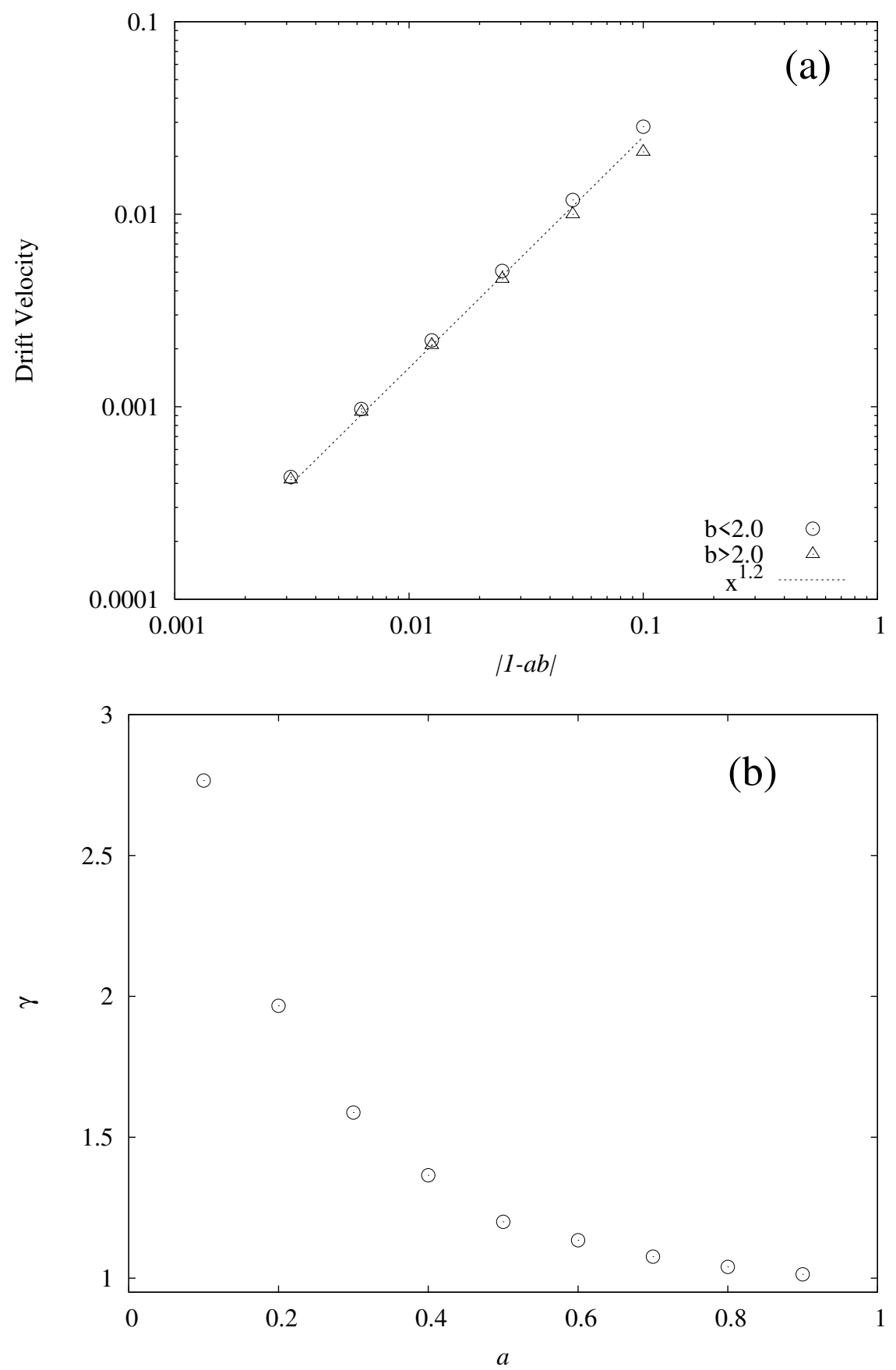

Figure 4: (a)Plot of the converged drift velocity for the PF model in the limit as $L \rightarrow \infty$ against $|1-a b|$, the degree of the breaking of the zero-current condition. (b)Plot of the exponent in Eq.(24) as a function of $a$. It converges to unity in the limit $a \rightarrow 1$. 


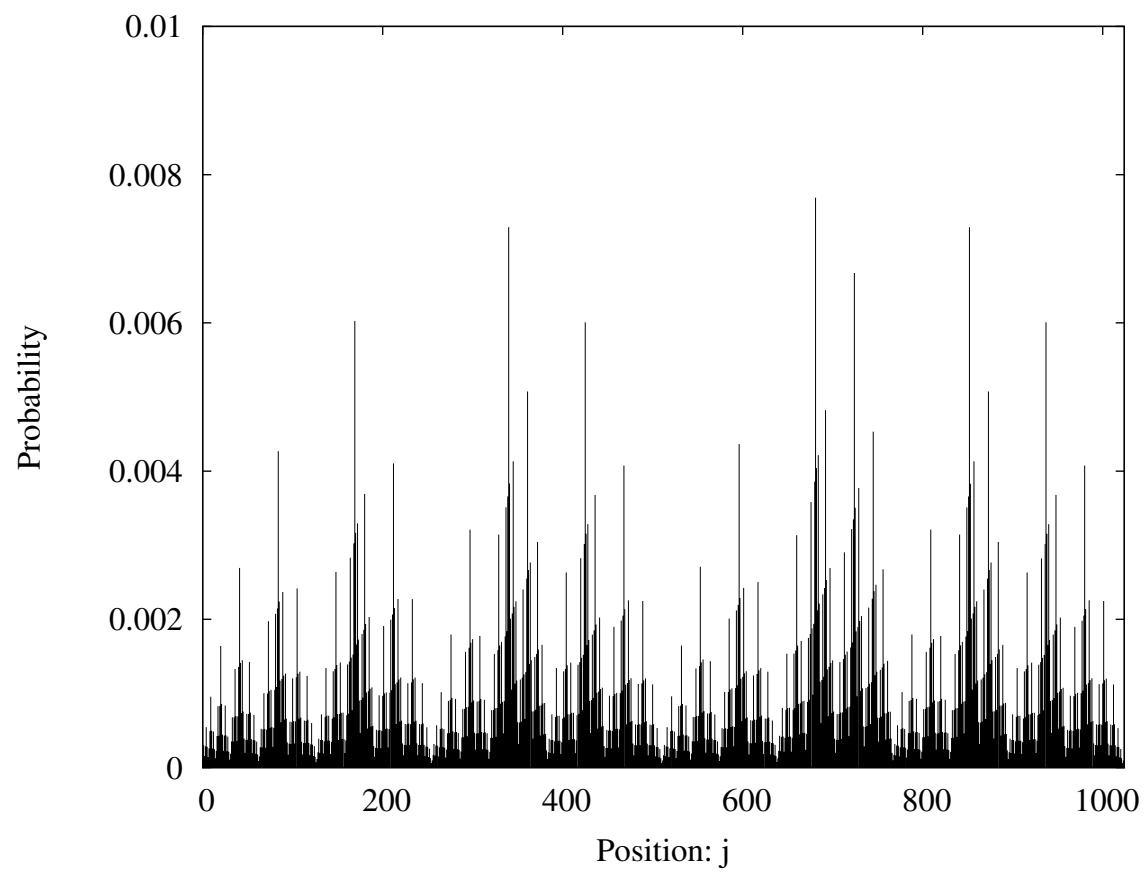

Figure 5: Stationary probability distribution for the PF model with $a=0.5, b=1.9$, and $L=1024$. The hierarchical structure of the distribution in the case without current is being broken and the distribution is being extended.

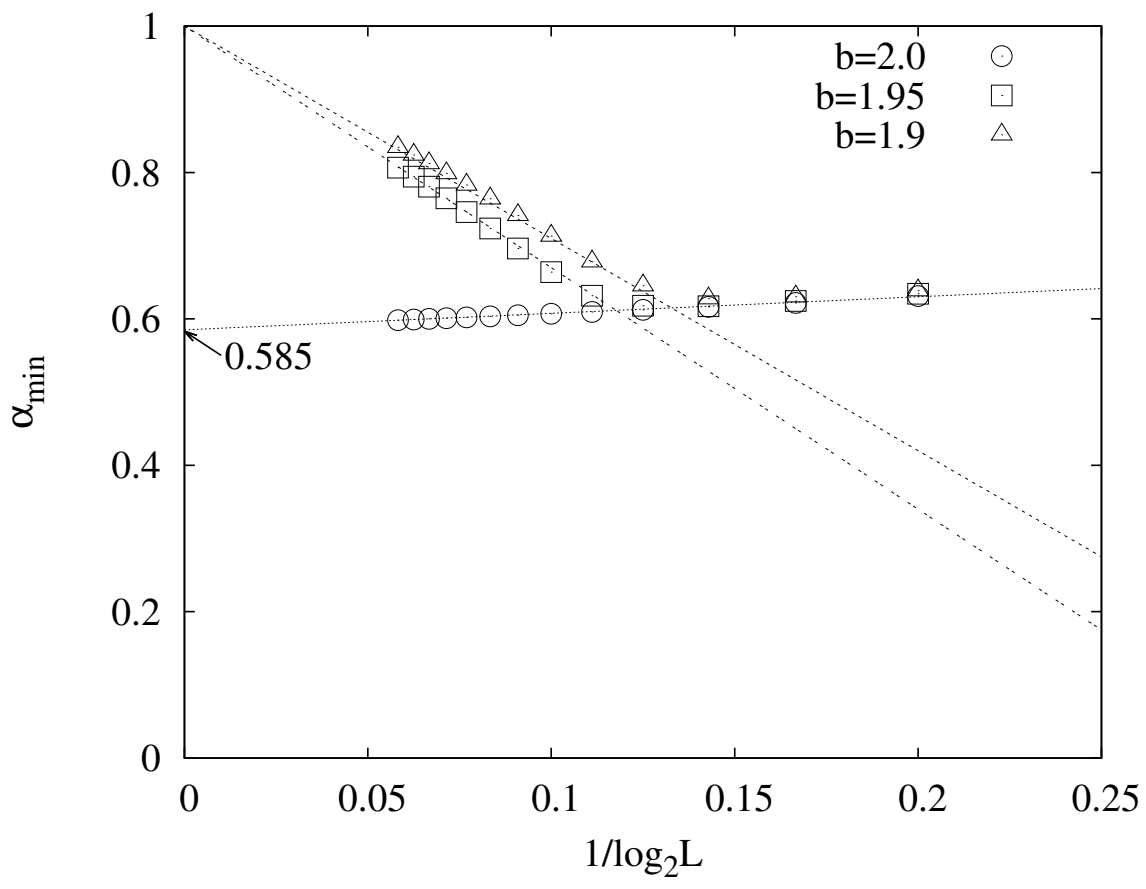

Figure 6: Plots of $\alpha_{\min }(L)$ against $1 / n=1 / \log _{2} L$ for the stationary distribution of the PF model with $a=0.5$ and several values of $b$. A shift is observed from the scaling behavior of a singular distribution to that of an extended distribution. 


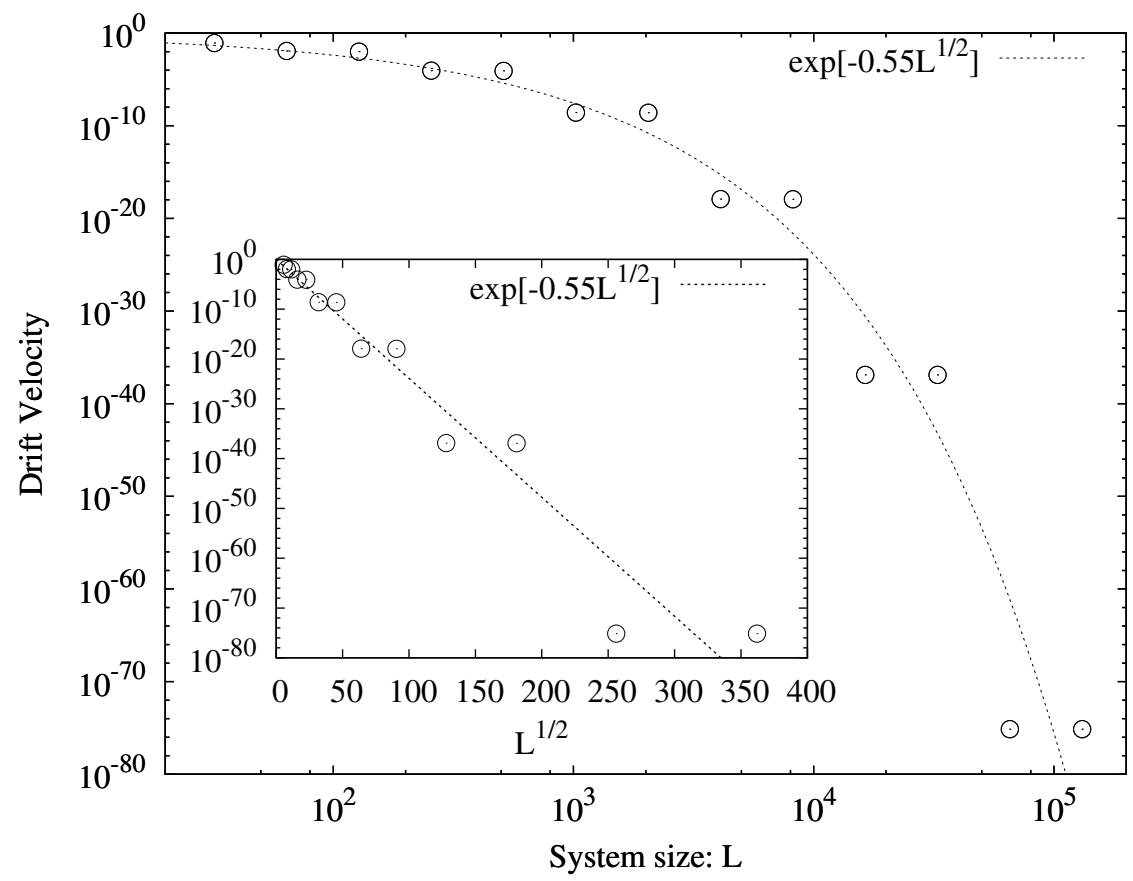

Figure 7: Dependence of the drift velocity of the RS model on the system size with $a=0.5$ and $b=2.0$. (Inset) The same plot with $\sqrt{L}$ as the abscissa.

$a=0.5, b=1.9$ and $L=1024$. It is observed that the hierarchical structure is gradually being broken and the distribution is forced to be extended.

Figure 6 6 shows the plots of $\alpha_{\min }(L)$ for several values of $b$ with $a=0.5$ fixed against $1 / n=1 / \log _{2} L$ for the stationary distribution. For $b=2.0$, the zero-current condition is satisfied in the infinite system size limit. The plot is linear and converges to a finite value, $\alpha_{\text {min }}=0.585$, in the limit as $\log _{2} L \rightarrow 0$ (i.e. $L \rightarrow \infty$ ). This means that the distribution is singular. Meanwhile, for $b=1.95$ and 1.9, where the zero-current condition does not hold, a different behavior is observed. For a small system, the plots are on a line corresponding to a singular distribution, as in the case with $b=2.0$. However, for a large system, the plot shifts to a line corresponding to an extended distribution, which converges to unity as $1 \log _{2} L \rightarrow 0$. The shift takes place at a smaller system size for $b=1.9$ than for $b=1.95$, since the degree of the breaking of the zero-current condition of the former is larger than that of the latter. In both cases, in the infinite system size limit, the stationary distribution is extended.

Note that the system size at which the shift takes place roughly corresponds to the size at which the drift velocity is saturated. For example, for $b=1.95$, the shift and saturation take place at $L \sim 10^{3}(n=9-10)$. This length is considered as a correlation length, within which the disorder is effective.

From these results, we can conclude that any small breaking of the zero-current condition induces nonzero drift velocity and forces the stationary distribution to be extended.

\subsection{Rudin-Shapiro(RS) model}

First we consider the case where the zero-current condition is satisfied in the infinite system size limit. Figure 7 shows the dependence of the drift velocity $v_{d}$ on the system size $L$ in the case with $a=0.5$ and $b=2.0$. It decays very rapidly as the system size increases and it is fitted well by

$$
v_{d}(L) \sim \exp \left[-c L^{1 / 2}\right]
$$

where $c \sim 0.55$ 


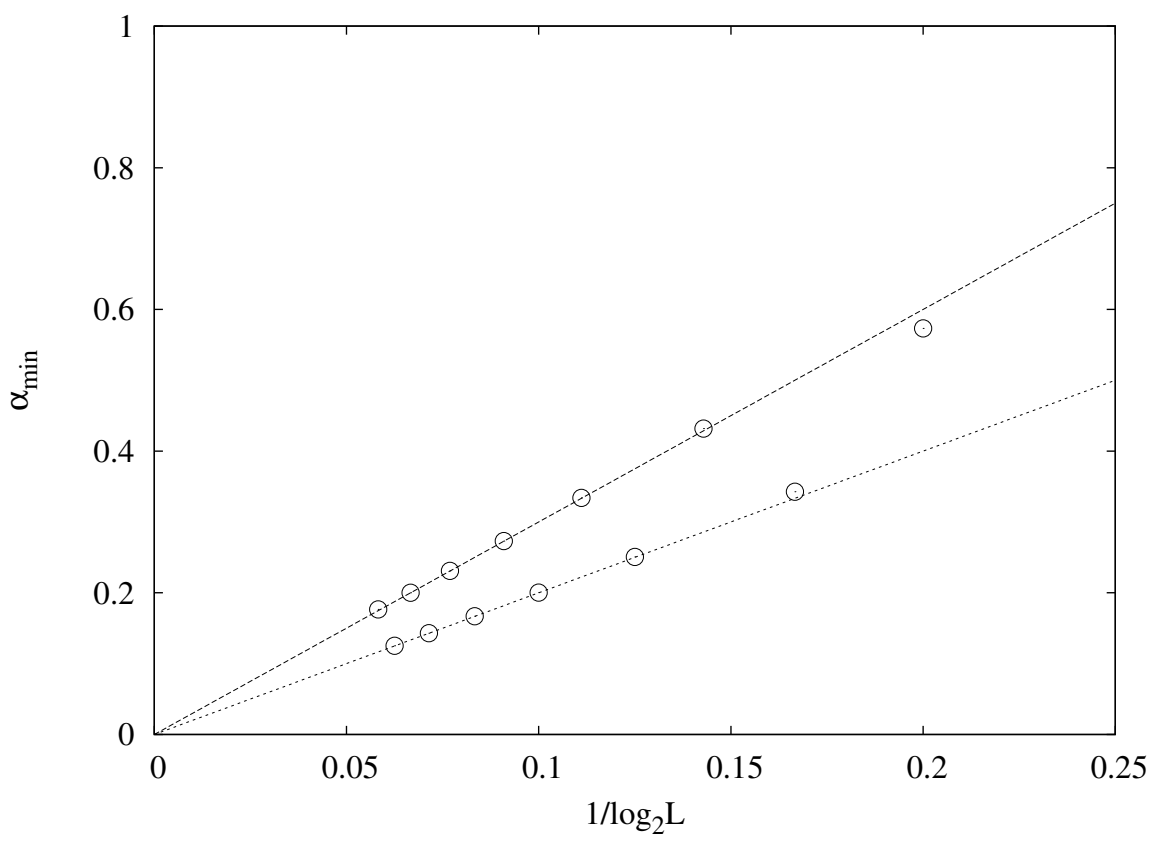

Figure 8: Plot of $\alpha_{\min }(L)$ against $1 / n=1 / \log _{2} L$ for the stationary distribution for the RS model with $a=0.5$ and $b=2.0$. The upper line corresponds to the group with odd $n$ and the lower to the group with even $n$.

Figure 8 shows the dependence of $\alpha_{\min }$ on $1 / n=1 / \log _{2} L$ for the stationary distribution in the same case. There is a parity dependence found, i.e. the plots are classified into two groups - of even $n$ and odd $n$. Anyway, although the plots in different groups are on different lines, both converges to zero in the infinite system size limit, $1 / \log _{2} L \rightarrow 0$. This fact indicates that the distribution is localized. This scaling behavior is a little different from that of the case where the zero-current condition is always satisfied in finite size lattice. In that case, the line corresponding to the group of odd $n$ is merged into that to the group of even $n$ for sufficiently large system size 11$]$.

Figure 9 shows the dependence of the drift velocity on the system size for several values of $b$ with $a=0.5$ fixed, where the zero-current condition does not hold. In these cases, if the system is small, as the system size increases, the drift velocity decreases. After that the system becomes larger than a certain threshold value, the drift velocity is saturated and converges to a certain finite value. For fixed $a$, as the degree of the breaking of the zero-current condition, $|1-a b|$, grows larger, the threshold value for the system size becomes smaller and the drift velocity converges to a larger value. However, unfortunately, it is quite difficult to obtain the value to which the drift velocity converges as a function of the degree of the breaking of the zero-current condition, since the smaller the degree of the breaking, the faster increases the threshold system size at which the drift velocity is saturated and converges, and the smaller the value to which the velocity converges.

Figure 10 shows the stationary probability distribution for $a=0.5, b=1.7$, and $L=1024$. Several high-density regions are observed and therefore the distribution is forced to be extended by a finite current.

Figure 11 shows the dependence of $\alpha_{\min }$ on $1 / n=1 / \log _{2} L$ for $a=0.5$ and $b=1.9$ and 1.7. For a small system, $\alpha_{\min }$ approaches zero as $1 / n \rightarrow 0$. This is the characteristic scaling behavior of a localized distribution, as in the case where the zero-current condition is satisfied in the infinite system size limit. Then, the plot shifts towards unity after the system becomes larger than a certain threshold value. This corresponds to the scaling behavior of an extended 


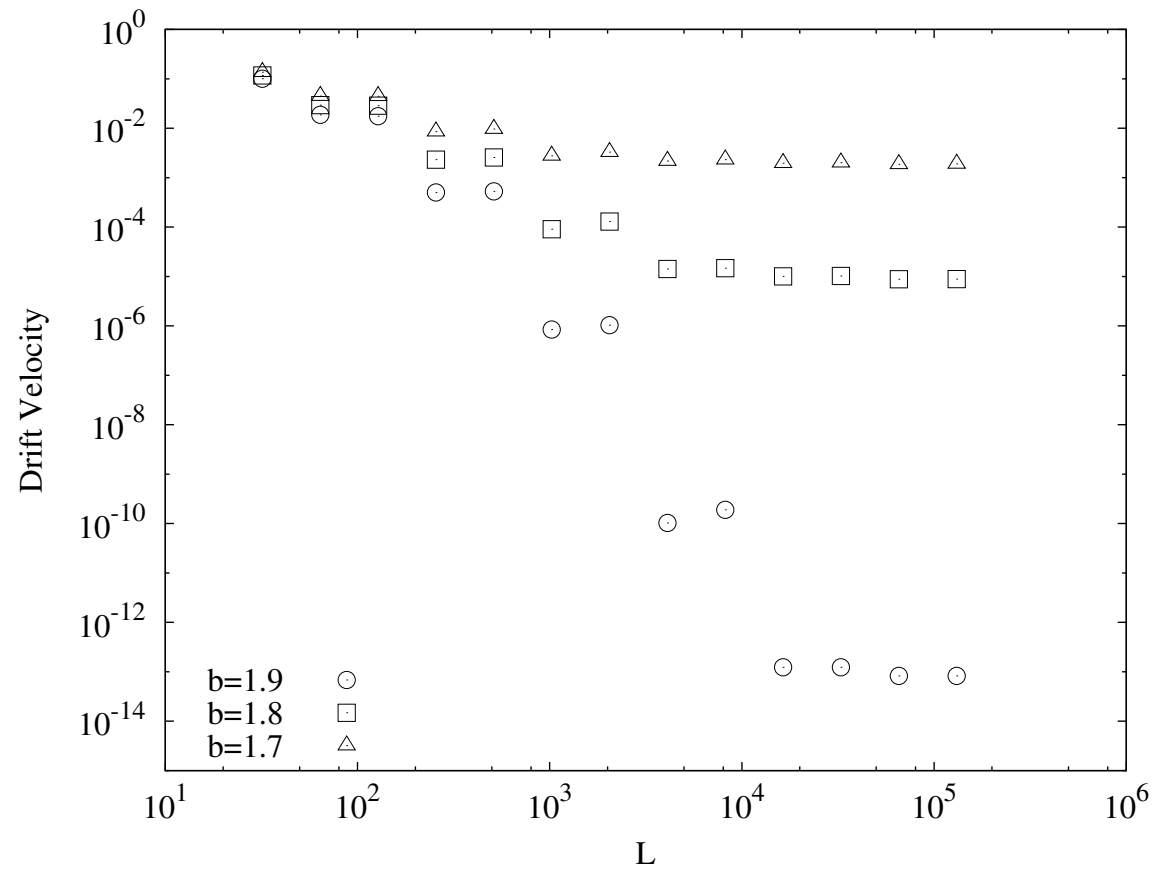

Figure 9: Plots of the drift velocity $v_{d}$ against the system size $L$ for the RS model with $a=0.5$ fixed and several values of $b$.

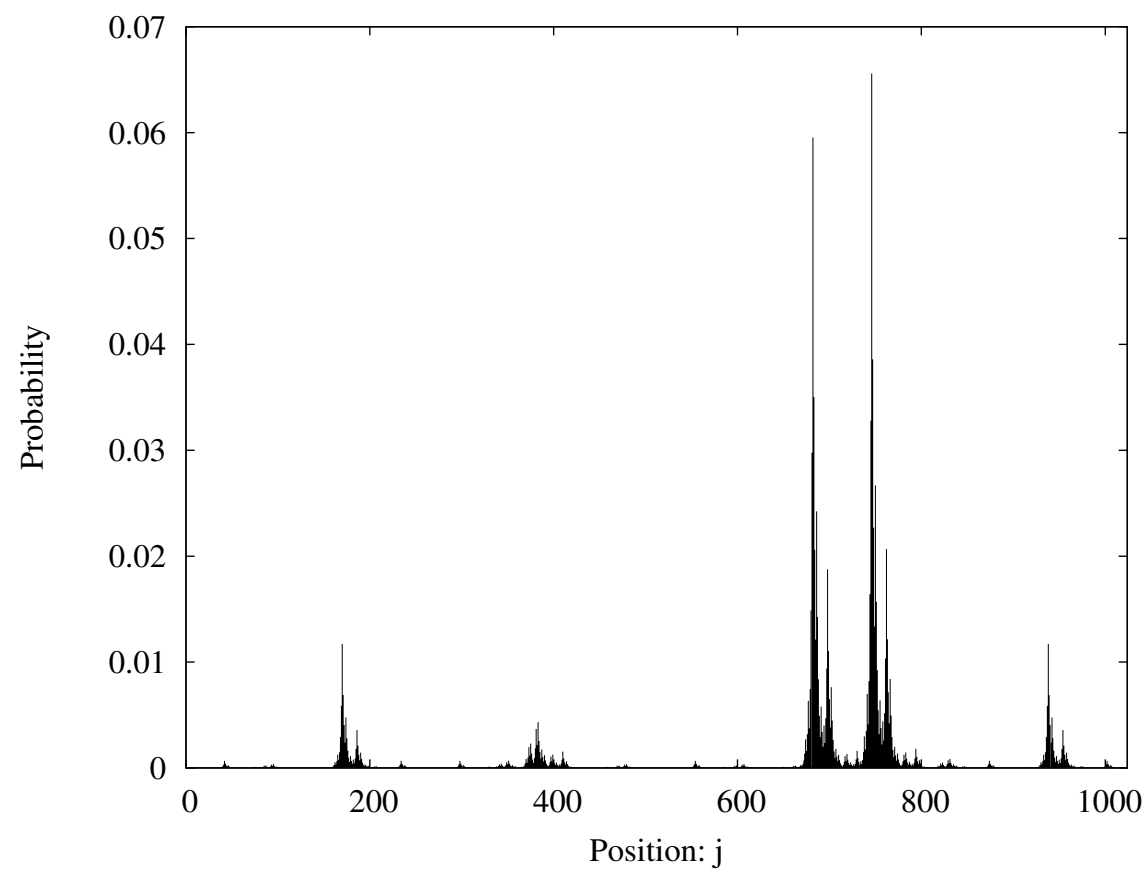

Figure 10: Stationary probability distribution for the RS model with $a=0.5, b=1.7$, and $L=1024$. Several high density regions can be observed. 


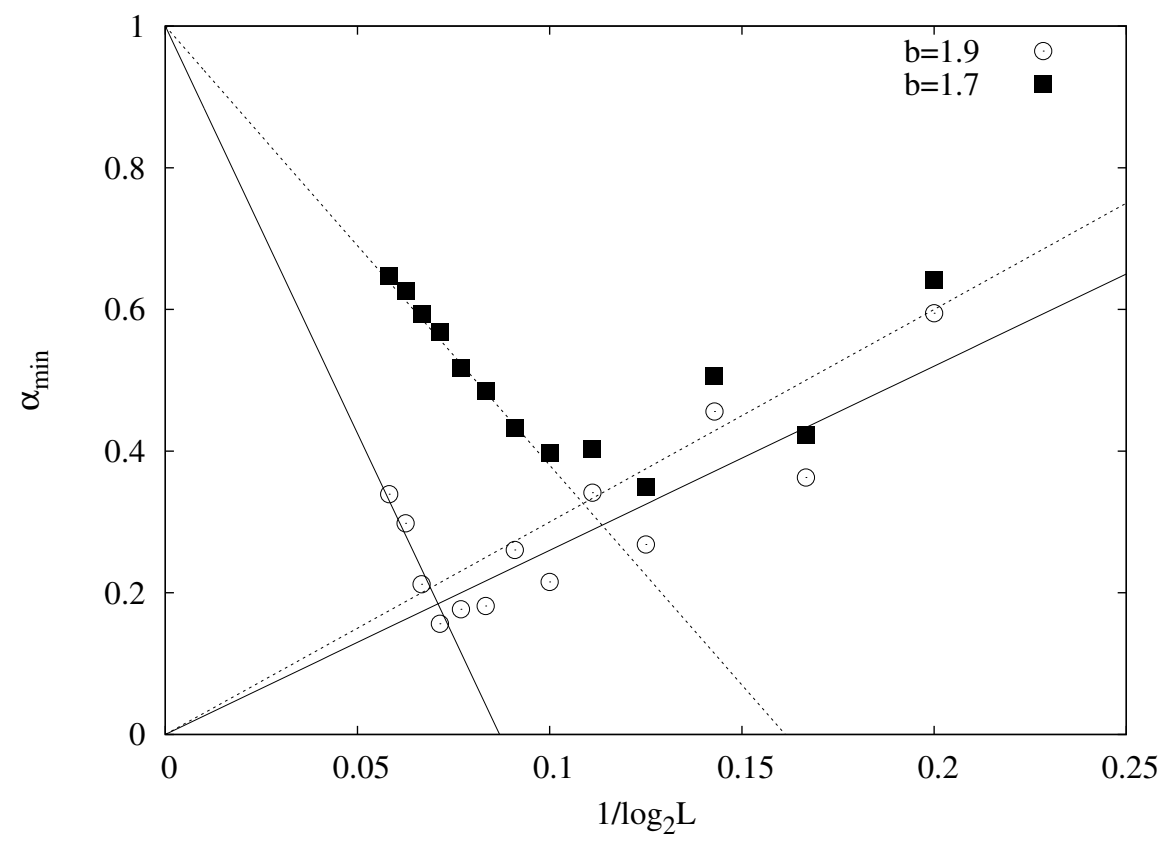

Figure 11: Plots of $\alpha_{\min }(L)$ against $1 / n=1 / \log _{2} L$ for the stationary distribution of the RS model with $a=0.5$ and $b=1.9$ and 1.7. A shift is observed from the scaling behavior of a localized distribution to that of an extended distribution.

distribution. Note that, as seen in the PF model, the system size at which the shift of the scaling behavior of $\alpha_{\min }$ takes place is roughly the same as that at which the saturation of the drift velocity begins, and the larger the degree of the breaking of the zero-current condition becomes, the smaller the threshold for the system size is.

It is known that, for a system with random binary disorder, the drift velocity may vanish in the infinite system size limit, even if the zero-current condition is not satisfied [4, 8, 9]. The drift velocity vanishes if (1) the ratio of the two symbols does not converge to unity and (2) there is a sufficiently large weight corresponding to the symbol of fewer occurences. Since the wandering exponent of the RS sequence coincides with that of a random binary sequence, we conclude that in the RS model a finite drift velocity is induced by any small breaking of the zero-current condition and the drift velocity breaks the localized distribution when the system is large.

\section{Conclusion and outlook}

We have investigated the stationary states of random walks on one-dimensional lattices with aperiodic disorder, where a finite current flows through the system. Binary aperiodic sequences were used to construct the disorder, where the ratio of the number of As to that of Bs converges to unity in the limit of infinite sequence length. We concluded that no matter how little the zero-current condition is broken in the limit of infinite system size, a finite drift velocity is induced and for a large system the stationary distribution is extended, irrespective of the wandering exponent of the underlying aperiodic sequence, which affects the diffusional behavior. This conclusion may sound natural, or even trivial, since irrespective of the property of the diffusion - normal, anomalous or ultraslow - it is well known that diffusion is dominant in small systems and drift is dominant in large systems. However, for randomly disordered cases, it has been known that under certain conditions, the drift velocity vanishes, even though the zero-current condition is broken in the infinite system size limit, if the ratio of the number of 
As to that of Bs does not converge to unity [4, 9]. In this sense, The symmetry between A and $\mathrm{B}$ is a special condition. It has not yet been clear how the drift velocity and the stationary distribution behave in cases with asymmetrical aperiodic disorder. We expect that the differences between random disorder and aperiodic disorder may be much clearer in those cases. This is a problems to be investigated in the future.

\section{Acknowledgments}

This research was supported by the initiative-based project E-05 "Creation and Sustainable Governance of New Commons through Foundation of Integrated Local Environmental Knowledge (ILEK)", Research Institute for Hunanity and Nature (RIHN).

\section{References}

[1] N.G.van Kampen, Stochastic processes in Physics and Chemistry, 3rd ed. North-Holland, 2007.

[2] R.B.Schinazi, Classical and spatial stochastic processes, Birkhäuser, 1999.

[3] S.Alexander, J.Bernasconi, W.R.Schneider, and R.Orbach, Excitation dynamics in random one-dimensional systems, Rev.Mod.Phys. 53(1981)175-198.

[4] J.-P.Bouchard and A.Georges, Anomalous diffusion in disordered media: Statistical mechanisms, models, and physical applications, Physics Reports 195(1990)127-293.

[5] P.Le Doussal, C.Monthus, and D.S.Fisher, Random walks in one-dimensional environments: Exact renormalization group analysis, Phys.Rev.E 59(1999)4795-4840.

[6] F.Iglói and C.Monthus, Strong disprder RG approach of random systems, Physics Reports 412(2005)277-431.

[7] F.Iglói, L.Turban and H.Rieger, Anomarous diffusion in aperiodic environments, Phys.Rev.E 59(1999)1465-1474.

[8] Y.G.Sinai, Limit behaviour of one-dimensional random walks in random environments, Theor. Probab. Appl. 27(1982)256-268.

[9] B.Derrida, Velocity and diffusion constant of a periodic one-dimensional hopping model, J.Stat.Phys.31(1983)433-450.

[10] L.Dal Negro, J.H.Yi.V.Nguyen, Y.Yi.J.Michel and L.C.Kimerling, Spectrally enhances light emission from aperiodic photonic structures, Appl.Phys.Lett. 86(2005)261905; V.Passias, N.V.Valappil, Z.Shi, L.Deych, A.A.Lisyansky and V.M.Menon, Luminescence properties of a Fibonacci photonic quasicrystal, Opt.Exp.17(2009)6636-6642.

[11] H.Miki, Scaling analysis of stationary probability distributions of random walks on onedimensional lattices with aperiodic disorder, Phys.Rev.E 89(2014)062105.

[12] T.C.Halsey, M.H.Jensen, L.P.Kadanoff, I.Procaccia, and B.I.Shraiman, Fractal measures and their singularities: the characterization of strange sets, Phys.Rev.A 33(1986)11411151.

[13] B.Chhabra and R.V.Jensen, Direct determination of the $f(\alpha)$ singularity spectrum, Phys.Rev.Lett.62(1989)1327-1330.

[14] B.Chhabra, C.Meneveau, R.V.Jensen and K.R.Sreenivasan, Direct determination of the $f(\alpha)$ singularity spectrum and its application to fully developed tuebulence, Phys.Rev.A 40(1989)5284-5294.

[15] H.Miki and H.Honjo, Multifractal distribution of dendrite on one-dimensional support, J.Phys.Soc.Jpn. 82(2013)034002. 
[16] H.Hiramoto and M.Kohmoto, Electronic spectral and wavefunction properties of onedimensional quasiperiodic systems: a scaling approach, Int.J.Mod.Phys.B 6(1992)281-320, and references therein.

[17] J.M.Luck, Critical behavior of the aperiodic quantum Ising chain in a transverse magnetic field, J.Stat.Phys. 72(1993)417-458.

[18] J.Hermisson, Aperiodic and correlated disorder in XY chains: exact results, J.Phys.A $33(2000) 57-80$.

[19] J.Ma and J.Holdener, When Thue-Morse meets Koch, Fractals 13(2005)191-206.

[20] M.Dekking, Paperfolding morphisms, plane-filling curves, and fractal tiles, Theor.Comput.Sci. 414(2012)20-37. 